\title{
Vitamin D Status is Independently Associated with Insulin Resistance in Patients with Type 2 Diabetes Mellitus
}

This article was published in the following Dove Press journal: Risk Management and Healthcare Policy

\author{
Hang Zhao (D) \\ Yong Tang' \\ Chong Zheng ${ }^{2}$ \\ Luping Ren ${ }^{1} * *$ \\ Guangyao Song ${ }^{1, *}$ \\ 'Endocrinology Department, Hebei \\ General Hospital, Shijiazhuang, Hebei, \\ 05005I, People's Republic of China; \\ ${ }^{2}$ Pediatric Orthopaedics, Shijiazhuang the \\ Third Hospital, Shijiazhuang, Hebei, \\ 0500I I, People's Republic of China
}

*These authors contributed equally to this work

Correspondence: Luping Ren; Guangyao Song

348, Heping West Road, Shijiazhuang, Hebei, 05005I, People's Republic of China Tel +863II85988975; +863II85988556 Email renlupingII22@163.com; sguangyao2@163.com
Purpose: This study aimed to examine whether 25-hydroxyvitamin D (25OHD) levels (an indicator of vitamin D status) are independently associated with insulin resistance (IR) in patients with type 2 diabetes mellitus (T2DM).

Patients and Methods: This was a cross-sectional study. Participants with T2DM were recruited from the Department of Endocrinology in Hebei General Hospital according to inclusion and exclusion criteria. Data on basic characteristics and blood parameters were collected. We used the IR index (20/[fasting C-peptide $\times$ fasting plasma glucose]) to evaluate IR. Potential confounding factors were selected from comparisons among different IR index groups of quartiles and were adjusted in different models.

Results: We included 172 subjects (121 men and 51 women) whose mean age was 53.2 \pm 10.6 years. Body mass index (BMI), DM course, insulin use, glycated hemoglobin (HbA1c), fasting blood glucose, fasting C-peptide, triglycerides (TG), high-density lipoprotein cholesterol (HDL-C), apolipoprotein A1 (ApoA1), and albumin were differed among different IR-index groups (all $\mathrm{P}$ value $<0.05$ ). In models 1 and 2, no or some confounding factors were adjusted for, and we found that there was no relationship between 25OHD and the IR index. In model 3, when all confounding factors (DM course, insulin use, BMI, HbA1c, TG, HDL-C, ApoA1, albumin and other bone turnover markers) were adjusted for, the IR index was increased by $5.6 \%$ when $25 \mathrm{OHD}$ levels increased by $1 \mathrm{ng} / \mathrm{mL}$ (odds ratio: 1.056; $95 \%$ confidence interval: $1.009,1.105$ ).

Conclusion: Vitamin D is independently associated with IR in patients with T2DM.

Keywords: diabetes mellitus, type 2, vitamin D, insulin resistance

\section{Introduction}

Insulin resistance (IR) or decreased insulin sensitivity refers to the inability of insulin to effectively promote glucose uptake by peripheral tissues (eg, skeletal muscle and adipose tissue) and inhibit liver glucose output. If insulin can produce enough insulin to compensate for IR, blood glucose levels can be maintained at a normal level. However, if pancreatic function is insufficient to allow for IR, blood glucose levels rise and diabetes mellitus (DM) gradually develop. IR is a compensatory response mechanism of the body to excess energy. In the early stage of type 2 diabetes mellitus (T2DM), its main feature is IR, and IR is the central link in the onset and progression of T2DM. ${ }^{1}$ Therefore, evaluation of IR is important.

With regard to the evaluation method of IR, the gold standard is the euglycemic-hyperinsulinemic clamp, and others include the insulin suppression test, ${ }^{2}$ 
homeostasis model assessment-IR, ${ }^{3}$ and the quantitative insulin sensitivity check index. ${ }^{4}$ Because of the difficulty in technical use of these methods, their overall complexity, and the nature of the population, their clinical applications are relatively limited. In recent years, researchers have continuously proposed new methods for evaluating IR, which not only ensure the accuracy but also ensure the universality of clinical applications. In our research, we adopted the new formula proposed by Ohkura et $\mathrm{al}^{5}$ who found that it is a simple and effective method of determining IR.

Recently, calcium and vitamin D were found to be related to IR and T2DM, but few studies have determined whether vitamin D is independently associated with IR. Therefore, the current study aimed to examine whether 25hydroxyvitamin D (25OHD), as an indicator of vitamin D status, is independently associated with IR in patients with T2DM.

\section{Patients and Methods Study Protocol}

This was a cross-sectional study and was registered in Chinese Clinical Trial Registry (Registration Number: ChiCTR2000029391).

The primary outcome was the association of 25OHD with IR as evaluated by the following equation: IR index = 20/(fasting C-peptide $\times$ fasting plasma glucose). This association was adjusted for different confounding factors. The secondary outcome was differences among different IR levels.

This study protocol was approved by Hebei General Hospital Ethics Committee and the study was in accordance with the Declaration of Helsinki. The researchers explained the study purpose to the participants and recruited patients signed informed consent forms. We kept the participants' information confidential.

\section{Subjects}

Participants were recruited from inpatients of the Department of Endocrinology in Hebei General Hospital. Male and female participants were aged $\geq 18$ years old. Inclusion criteria were as follows: (i) T2DM as diagnosed according to the definition of the World Health Organization and (ii) complete data. Exclusion criteria were as follows: (i) other types of DM, such as type 1 diabetes, gestational diabetes, and other special types of DM; (ii) incomplete data; (iii) patients with severe diseases, such as malignancy, myocardial infarction, cerebral infarction, renal failure, and heart failure; (iv) infectious diseases, such as pneumonia, urinary infection, and diabetic foot infection; and (v) acute complications of DM, such as diabetic ketoacidosis, diabetic hyperosmolar syndrome, and hypoglycemic coma.

\section{Assessment}

Basic characteristics of the participants, including age, sex, family history of DM, course of DM, alcohol drinking, cigarette smoking, and insulin use, were collected by questionnaires. Anthropometric values (body weight, height) were measured by one researcher. Levels of fasting blood glucose (FBG), fasting C-peptide (FCP), glycated hemoglobin (HbA1c), the serum lipid profile (triglycerides [TG], total cholesterol, high-density lipoprotein cholesterol [HDL-C], low-density lipoprotein cholesterol, very low-density lipoprotein cholesterol, apolipoprotein A1 [ApoA1], and apolipoprotein B), bone turnover markers (25OHD, $\beta$-C-terminal cross-linked telopeptide of type I collagen $[\beta-\mathrm{CTX}]$, osteocalcin $[\mathrm{OC}]$, procollagen type 1 $\mathrm{N}$-terminal propeptide [P1NP], and parathyroid hormone $[\mathrm{PTH}])$, total protein, and albumin were measured after 12$\mathrm{h}$ overnight fasting (Note: LDL-C levels were measured directly). Body mass index (BMI) was calculated as body weight $(\mathrm{kg}) /$ height $^{2}\left(\mathrm{~m}^{2}\right)$.

\section{Statistical Analyses}

All statistical analyses were performed by SPSS 22.0 (IBM, Inc., Armonk, NY, USA). The IR index was divided into four groups of quartiles. For continuous variables, ANOVA (normally distributed data, expressed as mean $\pm \mathrm{SD}$ ) and a nonparametric test (Kruskal-Wallis test) (data with a skewed distribution, expressed as median, 25th, 75th percentiles) were used to compare differences among different groups. For categorical variables, the Chi-Square test was used for comparisons (expressed as number and percentage). After comparisons, several factors were selected on the basis of $P<0.1$ and the clinical background. Ordinal regression analysis was used to examine the relationship of $25 \mathrm{OHD}$ and the IR index. Different models (models 1, 2, and 3) had adjustment for different confounding factors. The results are shown as odds ratios (ORs) and $95 \%$ confidence intervals (CIs). $P<0.05$ was considered as statistically significant. 


\section{Results}

\section{Study Population}

A total of 212 subjects with T2DM were recruited from the Department of Endocrinology. Thirty-one patients with infectious diseases, acute complications of DM, and severe diseases were excluded. Nine patients with incomplete data were also excluded. Finally, 172 subjects were included in the current study (Flow diagram shown in Figure 1). There were 121 male and 51 female subjects whose mean age was $53.2 \pm 10.6$ years. The median FBG level was $6.53 \mathrm{mmol} / \mathrm{L}$ $(5.69,7.88)$. The $25 \mathrm{OHD}$ level ranged from 4.37 to $45.14 \mathrm{ng} /$ $\mathrm{mL}$. General characteristics of subjects are shown in Table 1.

\section{Comparisons of Clinical and Biochemical Characteristics of All Subjects}

The IR index was divided into four groups (Q1, Q2, Q3 and $\mathrm{Q} 4)$. The course of T2DM differed among the different IR groups. In the $\mathrm{Q} 1$ group $(\mathrm{P}=0.003)$, $\mathrm{BMI}(P<0.001)$ and FBG $(P<0.001)$ levels were significantly higher compared with the other three groups. FCP levels in the groups increased in the order of $\mathrm{Q} 1>\mathrm{Q} 2>\mathrm{Q} 3>\mathrm{Q} 4(P<0.001)$. TG levels in the $\mathrm{Q} 1$ group were higher than those in the $\mathrm{Q} 4$ group ( $P=0.032$ ). HDL-C levels were higher in the $\mathrm{Q} 3$ and Q4 groups compared with the Q1 group $(P=0.002)$. ApoA1 levels in the Q2 and Q4 groups were higher than those in the Q1 group ( $P=0.008)$. HbAlc was significant for comparisons between groups, but there was no difference when compared within groups $(P=0.031)$ (Table 2$)$.

\section{Association of 25OHD and the IR Index} BMI, DM course, insulin use, HbA1c, FBG, FCP, TG, HDL-C, ApoA1, and albumin were selected for further analysis on the basis of the finding that they showed $P<0.1$ in the above-mentioned analyses. On the basis of clinical perspectives, bone turnover markers were also selected as confounding factors.

Model 1 was a crude model with no adjustment for potential confounding factors. We found that there was no relationship between 25OHD and the IR index (OR: 1.026; 95\% CI: $0.989,1.064)$. In model 2, there was adjustment for DM course, insulin use, BMI, HbA1c, TG, HDL-C, ApoA1, and albumin. We found no association between 25OHD and the IR index in model 2 (OR: $1.041 ; 95 \%$ CI: $0.998,1.085)$. In model 3 , there was adjustment for all confounding factors, including DM course, insulin use, BMI, HbA1c, TG, HDL-C, ApoA1, albumin, $\beta$-CTX, OC, P1NP, and PTH. We found that, when $25 \mathrm{OHD}$ levels increased by $1 \mathrm{ng} / \mathrm{mL}$, the IR index was increased by 5.6\% (OR: 1.056; 95\% CI: 1.009 , $1.105)$, which indicated that $25 \mathrm{OHD}$ was independently associated with IR (Table 3).

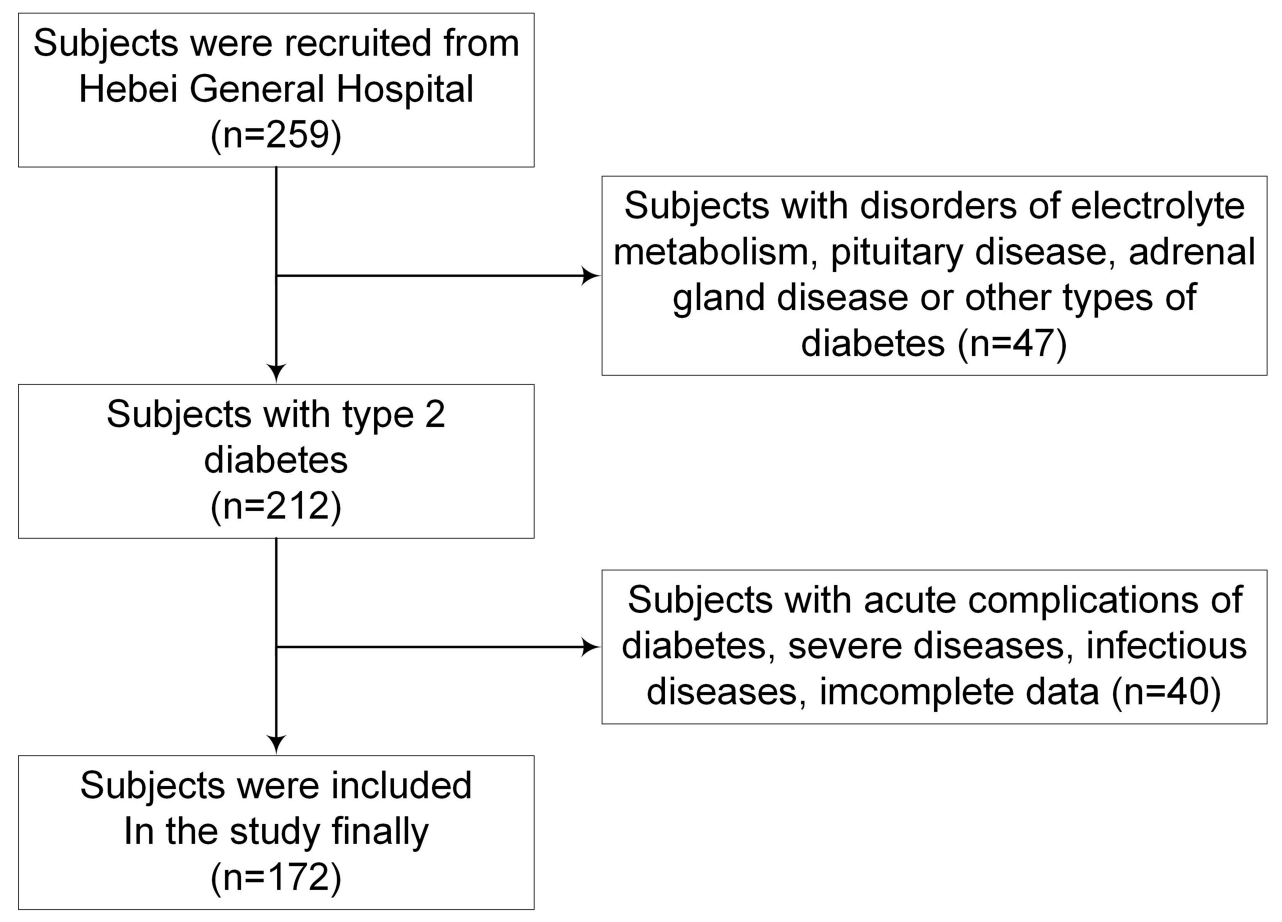

Figure I Flow diagram of recruitment. 
Table I General Characteristics of Subjects in Current Study

\begin{tabular}{|c|c|}
\hline & All Participants $(n=\mid 72)$ \\
\hline Male (n, \%) & 121 (70.3\%) \\
\hline Age (years) & $53.2 \pm 10.6$ \\
\hline Family history of DM & 61 (35.5\%) \\
\hline \multicolumn{2}{|l|}{ DM course (years) } \\
\hline $0-1$ & $32(18.6 \%)$ \\
\hline $1-10$ & 75 (43.6\%) \\
\hline $10-20$ & 49 (28.5\%) \\
\hline$\geq 20$ & $16(9.3 \%)$ \\
\hline History of hypertension & 72 (4I.9\%) \\
\hline Cigarette smoking & 65 (37.8\%) \\
\hline Alcohol drinking & $53(30.8 \%)$ \\
\hline Insulin use & $53(30.8 \%)$ \\
\hline BMI $\left(\mathrm{kg} / \mathrm{m}^{2}\right)$ & $25.77 \pm 3.31$ \\
\hline HbAlc (\%) & $9.1(7.5,10.8)$ \\
\hline $\mathrm{FBG}(\mathrm{mmol} / \mathrm{L})$ & $6.53(5.69,7.88)$ \\
\hline $\mathrm{FCP}(\mathrm{ng} / \mathrm{mL})$ & $1.69(1.16,2.48)$ \\
\hline $\mathrm{TC}(\mathrm{mmol} / \mathrm{L})$ & $4.65(4.05,5.45)$ \\
\hline TG (mmol/L) & $1.47(0.99,2.26)$ \\
\hline $\mathrm{HDL}-\mathrm{C}(\mathrm{mmol} / \mathrm{L})$ & $1.05(0.90,1.18)$ \\
\hline LDL-C (mmol/L) & $3.18 \pm 0.83$ \\
\hline VLDL-C (mmol/L) & $0.50(0.33,0.68)$ \\
\hline ApoAl (mmol/L) & $1.23(1.12,1.38)$ \\
\hline ApoB (mmol/L) & $0.80(0.68,0.98)$ \\
\hline Total protein $(g / L)$ & $68.50(63.3,72.93)$ \\
\hline Albumin $(\mathrm{g} / \mathrm{L})$ & $41.63 \pm 2.84$ \\
\hline $25 \mathrm{OHD}(\mathrm{ng} / \mathrm{mL})$ & $18.15(13.36,23.85)$ \\
\hline$\beta-C T X(n g / m L)$ & $0.36(0.24,0.52)$ \\
\hline $\mathrm{OC}(\mathrm{ng} / \mathrm{mL})$ & $12.05(9.70,15.83)$ \\
\hline $\mathrm{PINP}(\mathrm{ng} / \mathrm{mL})$ & $37.88(29.40,51.17)$ \\
\hline PTH (pg/mL) & $36.35(25.67,47.17)$ \\
\hline IR-index & $4.99(3.52,8.02)$ \\
\hline
\end{tabular}

Notes: For continuous variables, normally distributed data were expressed as mean \pm SD and data with a skewed distribution were expressed as median (25th, 75th percentiles). For categorical variables, data were expressed as number and percentage. Abbreviations: DM, diabetes; BMI, body mass index; HbAlc, glycated hemoglobin; FBG, fasting blood glucose; FCP, fasting C-peptide; TC, total cholesterol; TG, triglycerides, HDL-C, high-density lipoprotein cholesterol; LDL-C, low-density lipoprotein cholesterol, VLDL-C, very low-density lipoprotein cholesterol; ApoAI, apolipoprotein AI; ApoB, apolipoprotein B; 25-hydroxy vitamin D, $25 \mathrm{OH}$; $\beta$-CTX, $\beta$-C-terminal cross-linked telopeptide of type I collagen; OC, osteocalcin; PINP, procollagen type I N-terminal propeptide; PTH, parathyroid hormone; IR: insulin resistance.

\section{Discussion}

T2DM is a metabolic disease characterized by IR and defective insulin secretion. An effective method for evaluating IR is essential. The pancreas secretes insulin and C-peptide at a 1:1 ratio, but unlike insulin, C-peptide is not considerably cleared by the liver. Additionally, at physiological and supraphysiological concentrations, the kinetics of C-peptide are linear, resulting in a longer half-life. ${ }^{6,7}$ Therefore, peripheral C-peptide levels can reflect pancreatic insulin secretion more accurately compared with insulin. However, C-peptide is not restricted by using exogenous insulin and can be widely used in patients with T2DM.

Recent studies have found that an imbalance of calcium and vitamin D levels is related to IR, dysfunction of the pancreas, metabolic syndrome, and DM. Therefore, we focused on that determining whether vitamin D status is independently associated with IR.

In our study, according to quartiles, the IR index was divided into four groups. We found that the course of T2DM, BMI, glucose control (FBG, HbAlc) and FCP levels, and blood lipid (TG, HDL-C, ApoA1) levels were different among the groups. Because all $\mathrm{P}$ values were $<0.05$, these factors served as confounding factors to examine the relationship between IR and 25OHD. To avoid missing other confounding factors, we relaxed the screening criteria to 0.1 and clinical aspects were taken into account. Finally, BMI, DM course, insulin use, and levels of HbA1c, FBG, FCP, TG, HDL-C, ApoA1, albumin, and bone turnover markers were selected as confounding factors that may affect the results. Our previous study showed that a high BMI and TG levels protected against osteoporosis, and these patients tended to have higher vitamin D levels. ${ }^{8}$ Additionally, with extension of the course of DM, pancreatic function showed a downward trend, from the state of IR to the stage of insulin secretion disorder. Regression analysis showed that 25OHD and the IR index were not associated when we adjusted for no or some confounding factors. After adjusting for all potential confounding factors, we found that $25 \mathrm{OHD}$ and the IR index were independently related. The degree of IR decreased as 25OHD levels increased. These results are consistent with previous research results. Recent reviews that summarized a negative association between vitamin D deficiency and glycemic control showed a positive association between low vitamin D status and IR. ${ }^{9,10}$ In Arab American men, serum 25OHD levels are negatively correlated with homeostasis model assessment-IR, TG, FBG, and HbAlc levels. ${ }^{11}$ 
Table 2 Comparison of Parameters According to 20/(Fasting C-Peptide $\times$ Fasting Plasma Glucose) Quartiles

\begin{tabular}{|c|c|c|c|c|c|}
\hline & QI & Q2 & Q3 & Q4 & $P$ value \\
\hline Male (n, \%) & 32 (74.4\%) & 31 (72.1\%) & $30(69.8 \%)$ & $28(65.1 \%)$ & 0.333 \\
\hline Age (years) & $51.6 \pm 11.0$ & $51.8 \pm 9.2$ & $53.8 \pm 11.6$ & $55.6 \pm 10.2$ & 0.243 \\
\hline Family history of DM & 12 (27.9\%) & 17 (39.5\%) & 15 (34.9\%) & 17 (39.5\%) & 0.356 \\
\hline DM course (years) & & & & & 0.003 \\
\hline $0-1$ & 9 (20.9\%) & $8(18.6 \%)$ & 9 (20.9\%) & $6(14.0 \%)$ & \\
\hline $1-10$ & $22(51.2 \%)$ & $23(53.5 \%)$ & $16(37.2 \%)$ & 14 (32.6\%) & \\
\hline $10-20$ & $12(27.9 \%)$ & 8 (18.6\%) & $16(37.2 \%)$ & $13(30.2 \%)$ & \\
\hline$\geq 20$ & $0(0.0 \%)$ & $4(9.3 \%)$ & $2(4.7 \%)$ & $10(23.3 \%)$ & \\
\hline History of hypertension & 19 (44.2\%) & 17 (39.5\%) & 17 (39.5\%) & 19 (44.2\%) & 1.000 \\
\hline Cigarette smoking & 17 (39.5\%) & 17 (39.5\%) & 15 (34.9\%) & $16(37.2 \%)$ & 0.726 \\
\hline Alcohol drinking & 14 (32.6\%) & 14 (32.6\%) & II (25.6\%) & 14 (32.6\%) & 0.825 \\
\hline Insulin use & II (25.6\%) & 12 (27.9\%) & $10(23.3 \%)$ & $20(46.5 \%)$ & 0.066 \\
\hline BMI $\left(\mathrm{kg} / \mathrm{m}^{2}\right)$ & $27.53 \pm 3.06$ & $25.98 \pm 2.86$ & $25.19 \pm 3.33$ & $24.36 \pm 3.23$ & $<0.001$ \\
\hline $\mathrm{HbAlc}(\%)$ & $8.4(7.2,10.2)$ & $8.6(6.8,10.6)$ & $9.1(8.3,11.7)$ & $9.9(8.2,11.3)$ & 0.031 \\
\hline FBG (mmol/L) & $7.85(6.66,8.83)$ & $6.49(5.90,8.01)$ & $6.33(5.61,7.55)$ & $5.70(5.19,6.75)$ & $<0.001$ \\
\hline $\mathrm{FCP}(\mathrm{ng} / \mathrm{mL})$ & $3.02(2.49,3.36)$ & $2.09(\mathrm{I} .6 \mathrm{I}, 2.4 \mathrm{I})$ & $1.45(1.29,1.73)$ & $0.87(0.69,1.07)$ & $<0.001$ \\
\hline $\mathrm{TC}(\mathrm{mmol} / \mathrm{L})$ & $4.44(3.61,5.35)$ & $4.66(4.19,5.14)$ & $4.75(4.04,5.86)$ & $4.60(4.14,5.76)$ & 0.320 \\
\hline $\mathrm{TG}(\mathrm{mmol} / \mathrm{L})$ & $1.69(1.35,2.43)$ & $1.46(1.15,2.24)$ & $1.38(0.90,2.43)$ & I.32 (0.82, I.7I) & 0.032 \\
\hline HDL-C (mmol/L) & $0.95(0.84,1.05)$ & $1.04(0.92,1.20)$ & $1.07(0.90,1.24)$ & I.II $(0.93,1.25)$ & 0.002 \\
\hline LDL-C (mmol/L) & $2.99 \pm 0.92$ & $3.16 \pm 0.64$ & $3.33 \pm 0.82$ & $3.26 \pm 0.91$ & 0.305 \\
\hline VLDL-C & $0.50(0.33,0.68)$ & $0.46(0.35,0.66)$ & $0.50(0.32,0.65)$ & $0.52(0.34,0.72)$ & 0.990 \\
\hline ApoAl (mmol/L) & $1.18(1.06,1.24)$ & $1.24(1.13,1.44)$ & $1.21(1.13,1.44)$ & $1.30(1.19,1.39)$ & 0.008 \\
\hline ApoB (mmol/L) & $0.77(0.63,1.01)$ & $0.80(0.68,0.91)$ & $0.83(0.74,0.99)$ & $0.76(0.66,0.98)$ & 0.628 \\
\hline Total protein $(\mathrm{g} / \mathrm{L})$ & $68.60(63.20,73.30)$ & $66.80(63.30,73.10)$ & $69.90(63.30,74.20)$ & $68.50(63.90,72.30)$ & 0.894 \\
\hline Albumin $(g / L)$ & $42.28 \pm 2.62$ & $41.43 \pm 2.72$ & $42.03 \pm 3.26$ & $40.79 \pm 2.58$ & 0.068 \\
\hline $25 \mathrm{OHD}(\mathrm{ng} / \mathrm{mL})$ & I6.84 (II.86, 23.86) & I8.37 (14.48, 25.27) & $17.10(12.29,23.56)$ & $20.94(15.25,24.79)$ & 0.233 \\
\hline$\beta-C T X(n g / m L)$ & $0.31(0.24,0.47)$ & $0.43(0.24,0.57)$ & $0.31(0.22,0.52)$ & $0.35(0.27,0.54)$ & 0.610 \\
\hline$O C(n g / m L)$ & II.68 (9.7I, |4.95) & $12.59(10.00,17.61)$ & II.88 (9.54, I6.05) & I2.27 (9.64, I5.53) & 0.774 \\
\hline PINP (ng/mL) & $38.21(29.39,50.29)$ & $39.15(30.63,52.08)$ & $35.97(29.43,50.05)$ & $37.99(28.63,51.38)$ & 0.986 \\
\hline PTH $(\mathrm{pg} / \mathrm{mL})$ & $37.54(28.02,47.80)$ & $36.09(24.74,47.57)$ & $36.43(22.63,54.84)$ & $35.11(26.15,43.74)$ & 0.914 \\
\hline
\end{tabular}

Abbreviations: DM, diabetes; BMI, body mass index; HbAlc, glycated hemoglobin; FBG, fasting blood glucose; FCP, fasting C-peptide; TC, total cholesterol; TG, triglycerides, HDL-C, high-density lipoprotein cholesterol; LDL-C, low-density lipoprotein cholesterol, VLDL-C, very low-density lipoprotein cholesterol; ApoAI, apolipoprotein AI; ApoB, apolipoprotein B; 25-hydroxy vitamin D, 25OH; $\beta$-CTX, $\beta$-C-terminal cross-linked telopeptide of type I collagen; OC, osteocalcin; PINP, procollagen type I $\mathrm{N}$-terminal propeptide; PTH, parathyroid hormone; IR, insulin resistance.

Denova-Gutiérrez et al found an inverse relationship between IR and serum vitamin D levels was observed in 533 Mexican children and adolescents, approximately $90 \%$ of whom had sub-optimal vitamin D levels $(<30 \mathrm{ng} / \mathrm{mL}) .^{12}$
Our study provides clinicians with a simple method to assess IR and explore the relationship of IR with vitamin D status, suggesting the importance of vitamin D supplementation. Vitamin D deficiency is regarded as 
Table 3 Ordinal Regression Analysis of Association of 25OHD and IR

\begin{tabular}{|l|l|l|l|}
\hline & $\begin{array}{l}\text { Low } \\
\text { 25OHD }\end{array}$ & $\begin{array}{l}\text { Change of IR-Index Followed } \\
\text { by 25OHD Increases by } \\
\text { I ng/mL }\end{array}$ & P-value \\
\hline Model I & Reference & $1.026(0.989,1.064)$ & 0.171 \\
Model 2 & Reference & I.04I (0.998, I.085) & 0.059 \\
Model 3 & Reference & I.056 (I.009, I.105) & 0.020 \\
\hline
\end{tabular}

Notes: The results were showed as odds ratio and $95 \%$ confidence interval. Model I: no confounding factors were adjusted. Model 2: DM course, insulin use, BMI, HbAlc, TG, HDL-C, ApoAl and albumin were adjusted. Model 3: DM course, insulin use, BMI, HbAIc, TG, HDL-C, ApoAI, albumin, $\beta$-CTX, OC, PINP and PTH were adjusted.

Abbreviations: DM, diabetes; BMI, body mass index; HbAlc, glycated hemoglobin; TG, triglycerides, HDL-C, high-density lipoprotein cholesterol; ApoAI, apolipoprotein Al; 25-hydroxy vitamin D, $25 \mathrm{OH} ; \beta$-CTX, $\beta$-C-terminal cross-linked telopeptide of type I collagen; OC, osteocalcin; PINP, procollagen type I $\mathrm{N}$-terminal propeptide; PTH, parathyroid hormone; IR, insulin resistance.

a common health problem, which may affect up to half of the general population. ${ }^{13}$ A sedentary lifestyle for a long time, a lack of physical exercise, and minimal exposure to sunshine may account for the prevalence of vitamin D deficiency. ${ }^{14}$ Osteoporosis is an outcome of vitamin D deficiency ${ }^{15}$ and can lead to fracture and disability, which affect quality of life, and cause a burden to family and society. ${ }^{16}$ The Endocrine Society Guidelines recommend supplementation with 50,000 IU vitamin D3 once per week for 8 weeks. ${ }^{17}$ A meta-analysis of 11 randomized, controlled trails (total of 601 subjects) showed that continuous low doses of vitamin $\mathrm{D}(<4000 \mathrm{IU} / \mathrm{d})$ or supplementation with vitamin $\mathrm{D}$ as a co-supplement may improve insulin sensitivity in women with polycystic ovary syndrome. $^{18}$

The current study has several strengths. First, all potential confounding factors were adjusted for and 25OHD was independently associated with IR. Second, calculating the formula of IR was easy. The indicators used in this formula were common in our clinical work. Third, the evaluation method of IR in our study was non-invasive.

This study has some limitations. One of the limitations in our study is that the sample size was small for a crosssectional study. Another limitation is that only 10 subjects had vitamin D deficiency. Therefore, 250HD levels were not divided into three groups of normal vitamin D levels, vitamin D insufficiency, and vitamin D deficiency. We could not examine the relationship of 25OHD as a categorical variable and IR. From a clinical perspective, $25 \mathrm{OHD}$ as a categorical variable is more appropriate.

In conclusion, 25OHD is independently associated with IR in patients with T2DM. Proper supplementation of vitamin D in these patients may improve insulin sensitivity.

\section{Abbreviations}

ApoA1, apolipoprotein A1; ApoB, apolipoprotein B; BMI, body mass index; DM, diabetes mellitus; HbA1c, glycated hemoglobin; HDL-C, high-density lipoprotein cholesterol; IR, insulin resistance; LDL-C, low-density lipoprotein cholesterol; T2DM, type 2 diabetes mellitus; TC, total cholesterol; TG, triglyceride; VLDL-C, very low-density lipoprotein cholesterol; OC, osteocalcin; OR, odds ratio; P1NP, procollagen type 1 N-terminal propeptide; PTH, parathyroid hormone; 25OHD, 25-hydroxyvitamin D; CI, confidence interval; $\beta$-CTX, $\beta$-C-terminal cross-linked telopeptide of type I collagen.

\section{Data Sharing Statement}

The data can be obtained by email request (hangzhao4006@163.com).

\section{Ethics Approval}

The study was approved by the Ethics Committee of Hebei General Hospital.

\section{Acknowledgments}

We thank Ellen Knapp, PhD, from Liwen Bianji, Edanz Group China (www.liwenbianji.cn/ac), for editing the English text of a draft of this manuscript.

\section{Funding}

This study is not being supported by a grant form.

\section{Disclosure}

The authors report no conflicts of interest related to this work.

\section{References}

1. Boden G. Pathogenesis of type 2 diabetes. Insulin resistance. Endocrinol Metab Clin North Am. 2001;30(4):801-815. doi:10.1016/ S0889-8529(05)70216-4

2. Dahan MH, Abbasi F, Reaven G. Relationship between surrogate estimates and direct measurement of insulin resistance in women with polycystic ovary syndrome. J Endocrinol Invest. 2019;42 (8):987-993. doi:10.1007/s40618-019-01014-9

3. Fasipe QJ, Ayoade OG, Enikuomehin AC. Severity grade assessment classifications for both insulin resistance syndrome and status of pancreatic beta cell function in clinical practice using homeostasis model assessment method indices. Can J Diabetes. 2020;44(7):663-669. doi:10.1016/j.jcjd.2020.02.003

4. Yu Y, Pan F, Cui W, Tang W, Hu Y. Differences in early-phase insulin secretion and glucose disposition index between aged and middle-aged patients with newly diagnosed type 2 diabetes. Geriatr Gerontol Int. 2020;20(3):206-211. doi:10.1111/ggi.13861 
5. Ohkura T, Shiochi H, Fujioka Y, et al. 20/(fasting C-peptide $\times$ fasting plasma glucose) is a simple and effective index of insulin resistance in patients with type 2 diabetes mellitus: a preliminary report Cardiovasc Diabetol. 2013;12:21. doi:10.1186/1475-2840-12-21

6. Eaton RP, Allen RC, Schade DS, Erickson KM, Standefer J. Prehepatic insulin production in man: kinetic analysis using peripheral connecting peptide behavior. J Clin Endocrinol Metab. 1980;51 (3):520-528. doi:10.1210/jcem-51-3-520

7. Polonsky KS, Licinio-Paixao J, Given BD, et al. Use of biosynthetic human C-peptide in the measurement of insulin secretion rates in normal volunteers and type I diabetic patients. J Clin Invest. 1986;77 (1):98-105. doi:10.1172/JCI112308

8. Zhao H, Zheng C, Gan KX, et al. High body mass index and triglycerides help protect against osteoporosis in patients with type 2 diabetes mellitus. J Diabetes Res. 2020;2020:1517879. doi: $10.1155 / 2020 / 1517879$

9. Sacerdote A, Dave P, Lokshin V, Bahtiyar G. Type 2 diabetes mellitus, insulin resistance, and vitamin D. Curr Diab Rep. 2019;19 (10):101. doi:10.1007/s11892-019-1201-y

10. Lips P, Eekhoff M, Van Schoor N, et al. Vitamin D and type 2 diabetes. J Steroid Biochem Mol Biol. 2017;173:280-285. doi:10.1016/j.jsbmb.2016.11.021

11. Pinelli NR, Jaber LA, Brown MB, Herman WH. Serum 25-Hydroxy Vitamin D and insulin resistance, metabolic syndrome, and glucose intolerance among Arab Americans. Diabetes Care. 2010;33 (6):1373-1375. doi:10.2337/dc09-2199
12. Denova-Gutiérrez E, Muñoz-Aguirre P, López D. Low serum vitamin $\mathrm{D}$ concentrations are associated with insulin resistance in Mexican children and adolescents. Nutrients. 2019;11(9). doi:10.3390/ nu11092109

13. He C, Lin Z, Robb SW, Ezeamama AE. Serum vitamin D levels and polycystic ovary syndrome: a systematic review and meta-analysis. Nutrients. 2015;7(6):4555-4577. doi:10.3390/nu7064555

14. Holick MF. Vitamin D status: measurement, interpretation, and clinical application. Ann Epidemiol. 2009;19(2):73-78. doi:10.1016/j. annepidem.2007.12.001

15. Yang R, Chen J, Zhang J, et al. 1,25-Dihydroxyvitamin D protects against age-related osteoporosis by a novel VDR-Ezh2-p16 signal axis. Aging Cell. 2020;19(2):e13095. doi:10.1111/acel.13095

16. Gosch M, Bail HJ, Grueninger S, Stumpf U, Kammerlander C, Wicklein S. What is a reasonable rate for specific osteoporosis drug therapy in older fragility fracture patients? Arch Osteoporos. 2002;15 (1):20. doi:10.1007/s11657-020-0690-2

17. Holick MF, Binkley NC, Bischoff-Ferrari HA, et al. Evaluation, treatment, and prevention of Vitamin D deficiency: an Endocrine Society Clinical Practice Guideline. J Clin Endocrinol Metab. 2011;96(7):1911-1930. doi:10.1210/jc.2011-0385

18. Łagowska K, Bajerska J, Jamka M. The role of Vitamin D oral supplementation in insulin resistance in women with polycystic ovary syndrome: a systematic review and meta-analysis of randomized controlled trials. Nutrients. 2018;10(11). doi:10.3390/nu10111637
Risk Management and Healthcare Policy

\section{Publish your work in this journal}

Risk Management and Healthcare Policy is an international, peerreviewed, open access journal focusing on all aspects of public health, policy, and preventative measures to promote good health and improve morbidity and mortality in the population. The journal welcomes submitted papers covering original research, basic science, clinical \& epidemiological studies, reviews and evaluations, guidelines, expert opinion and commentary, case reports and extended reports. The manuscript management system is completely online and includes a very quick and fair peer-review system, which is all easy to use. Visit http://www.dovepress.com/testimonials.php to read real quotes from published authors. 